\title{
Comparison of accuracy between ultrasound B scan and partial coherence interferometry (IOL master) in IOL (intraocular lens) power calculation
}

\author{
Aditi Sharma ${ }^{1, *}$, Aditya Vikram Sharma ${ }^{2}$ \\ ${ }^{1,2}$ Consultant, Dept. of Ophthalmology, Kalyani Eye Hospital, Rajasthan, India \\ *Corresponding Author: Aditi Sharma \\ Email: jain.adt@gmail.com
}

\begin{abstract}
Aims and Objectives: To compare the accuracy of ultrasound A scan biometry and IOL master (PCI) biometry in intraocular power calculation, by doing a comparative study of difference in biometry by conventional methods and optical biometry (IOL master).

Materials and Methods: Place of study: Aravind Eye Hospital, India.

Design of Study: Prospective, non randomised, clinical study.

Study Group and Duration: The study involves patients who underwent cataract surgeries at Aravind Eye Hospital, Tirunelveli during the specified time period.

The IOL power and predicted refractive outcome were calculated for every patient by both the methods, A scan ultrasound Biometry IOL master (Partial Coherence interferometry)

Inclusion Criteria: Only those patients in whom it was possible to secure a reliable IOL master reading (on the basis of good SNR), were included.

Results: In a 12 month period, 337 eyes were consecutively enrolled in the study. Both ultrasound A scan and partial coherence interferometry was performed and IOL power implanted as per the IOL master.

Inclusion criterion and exclusion criterion used as mentioned.

The difference in between the final and predicted refraction is compared for both the groups. The overall Mean absolute error was $0.402 \pm 0.407$ with the ultrasound A scan \& $0.388 \pm 0.406$ for the IOL master, with the probability value ( $\mathrm{p}$-value) 0.3802 . The mean numerical error (the difference between the refractive outcome and the predicted spherical equivalent) is $-0.226 \pm 0.526$ (min $-2.66 \& \max 2.06$ ) for the ultrasound scan group and $-0.217 \pm 0.518$ (min $-2.41 \& \max 2.10$ ) for the IOL master group, with the probability value (p-value) being 0.514 .

We got a comparative result in our study, though not significant, but it is more in favour of IOL master (PCI) as per the mean absolute error and mean numerical error calculations.

When analysing the Mean Axial Length readings, the findings were almost similar, with an insignificant p-value.
\end{abstract}

Keywords: Biometry, IOL master, Power calculation, Ultrasound.

\section{Introduction}

Cataract surgery, the most frequently performed surgery in ophthalmology, has evolved so much in past few years that it has become more of a refractive surgery rather than a curative one.

There are no alternatives to the surgery, and the new advances aim to restore as normal a vision as possible. First step towards emmetropia in cataract surgery is accurate estimation of the IOL power.

The main variables in biometry are average corneal refractive power, anterior chamber depth (A-constant) and axial length of the eye, ${ }^{1}$ which can be computed by a variety of IOL calculation formulae. ${ }^{9-12}$

The accuracy of post cataract surgery refraction depends on minimal errors associated with these parameter measurements. It requires a skilled technician, optimal corneal surface contact and significant time duration.

Also errors associated with axial length have the highest impact on post operative refraction and it may account for more then half of deviation from the expected outcome following routine cataract surgery. ${ }^{1}$

Traditionally ultrasound (A-scan) is been the most commonly used methods for axial length measurement. ${ }^{1}$
An important drawback of this technique is the occurrence of corneal indentation caused by contact with ultrasound probe, thus shortening the eye and leading to an underestimation of the true axial length ${ }^{1,7}$ and shifting the post-operative refraction towards myopia.

With the introduction of non-contact optical biometry (Laser Interferometry) we have revolutionized post-operative IOL selection by eliminating these difficulties. ${ }^{9}$

One instrument - Intraocular lens master [IOLm]; Carl Zeiss Meditec, Jena, Germany. Which uses partial coherence interferometry, a non contact method, was introduced in $2000 .^{6}$

So, partial coherence laser interferometry has become the preferred instrument for calculating axial length. ${ }^{8,9}$

Many studies ${ }^{14-19}$ have been already conducted to compare the precision of the predictive value of the IOL master with that of the traditional ultrasound biometry. Recent publications have reported mixed conclusions about both the technology regarding post-operative refraction. ${ }^{6}$

In a prospective study by Gantenbein et al 162 consecutive eyes undergoing cataract surgeries, the 
authors concluded that contact axial biometry offered a better prediction of final refraction than the IOL master, but that the IOL master was an easier and faster tool to use. ${ }^{13}$ One published trial of small sample size reported no statistically significant difference in the postoperative refractive error in patients with laser interferometrycalculated IOLs implanted, compared with ultrasound biometry.

It is also known that laser interferometry is unable to measure IOL power in close to one thirds of patients because of dense cataracts, sub capsular opacities, or a signal/noise ratio less than $2: 1$. The inability of IOL master (Carl Zeiss Meditec, Inc., Dublin, CA) to predict IOL power in some eyes, all of which were able to be measured by ultrasound, has resulted in exclusion of patients adverse to PCI from comparison investigations, resulting in inconclusive clinical results and biased outcomes. ${ }^{13}$

Some authors also pointed out that in eyes with significant cataract, axial biometry was still needed for better axial length measurements. More recently it was suggested that the axial length measurements with IOL master were minimally affected by the cataract grade.

Two small case series have examined the effect of macular disease on both the techniques with a suggestion that the IOL master may be more accurate in these cases.

In this study, we have evaluated eyes posted for cataract surgery in a prospective fashion estimating the IOL power in the same patient with both traditional axial biometry and the IOL master.

\section{Aims and Objectives}

To determine the precision and accuracy of Ultrasound A scan biometry and IOL master (PCI) biometry in intraocular power calculation, by doing a comparative study.

\section{Materials and Methods}

Place of Study: Aravind Eye Hospital, Tirunelveli (Tamil Nadu), India

Design of Study: Prospective, Non Randomised, Clinical study.

Study group and Duration: The study involves patients who underwent cataract surgeries at Aravind Eye Hospital, Tirunelveli during the specified time period.

The IOL power and predicted refractive outcome were calculated for every patient by both the methods,

1. A scan Ultrasound Biometry

2. IOL master (Partial Coherence interferometry)

Inclusion Criteria: Only those patients in whom it was possible to secure a reliable IOL master reading (on the basis of good SNR), were included.

IOL power was implanted in-the-bag, as calculated by IOL master by a single surgeon in the specified time period.

A thorough pre-operative assessment was conducted for every patient.
1. Subjective refraction and best corrected visual acuity

2. Slit lamp biomicroscopic examination (corneal clarity, pupil examination, cataract type etc.),

3. Intraocular pressure (NCT or Goldmann's applanation tonometer)

4. Detailed fundus examination was done under dilatation (slit lamp $90 \mathrm{D}$ )

Post-operative uncorrected, best corrected visual acuity and slit lamp examination was done at $1^{\text {st }}$ day and $1^{\text {st }}$ month.

\section{Exclusion Criteria:}

Following were excluded form study.

1. Borderline, Low SNR cases.

2. Corneal pathology

3. Corneal curvature abnormality (previous penetrating karatoplasty or refractive procedures)

4. Central corneal opacity

5. Corneal dystrophy / degeneration

6. Eyes with dense cataracts (advanced/mature cataracts) and media opacities.

7. Keratoconjuctivitis sicca

8. Absence of direct pupillary reaction and RAPD

9. Lens induced glaucoma and angle closure glaucoma

10. Any retinal pathology like

a. Macular holes

b. Retinal vascular occlusions

c. Gross retinopathy changes due to diabetes or hypertension

d. Heredo-macular degeneration

11. Any previous trauma to eye

12. Eyes with prior ocular surgeries

13. Cases that had complication at the time of surgery.

14. Cases with cataract surgeries combined with another procedure

15. Amblyopic eye

16. Paediatric cases (age $<15$ years).

The IOL master (Carl Zeiss Humphrey Systems) was used for biometry in all the patients, following the manufacturer's recommendations.

This was followed by keratometry using the manual keratometer and A -Scan contact solid probe biometry using the ultrasound unit (Sonomed Inc.).

The same experienced biometry technician made both measurements.

The IOL power and predicted post-operative refraction with each IOL were determined by the SRK T formula.

The optical distances yielded by IOL master (PCI) were divided by the group refractive indices of the respective ocular media to obtain geometrical distances.

The values $1.3440,1.3454,1.3851$ ad 1.4065 were used for group refractive indices of vitreous, aqueous, cornea and the lens, respectively.

For the conversion of optical to geometrical values of lens thickness, a constant group refractive index for all cataract grades was assumed. 
For ultrasound biometry, sound velocities of 1532 $\mathrm{m} / \mathrm{s}$ were used for the aqueous and vitreous humour and $1641 \mathrm{~m} / \mathrm{s}$ for the lens.

A Minimum of 10 measurements was obtained for each parameter (etc total axial length) in each eye for calculating mean values. The total AL was obtained for both techniques by adding the means of the measured intraocular distances.

The SRK T formula combined with US biometry was used to calculate the desired IOL power, Preoperative PCI (IOL master) biometry data of the same 366 eyes combination with the 4 IOL power formulas $(2,108,109,110)$ were used to determine the refractive power of the IOLs in mentioned studies, but in this study we used SRK T.

All the patients underwent uneventful phacoemulsification through temporal clear corneal, self sealing, $3.0 \mathrm{~mm}$ incision, by a single surgeon.

Intraocular lenses were implanted in the bag and where of two types, Acrysof (Alcon Lab.) and Auro foldable (Aurolab)

Follow up was done at First day and 4 weeks postoperative.

\section{Statistical Analysis \\ Software}

STATA - Statistical software; version 8.1 (Stata Corp, College Station, TX)

Analysis

Wilcoxon rank-sum (Mann - Whitney) test was used for statistical Analysis.

A p-value less then $0.05(\mathrm{p}<0.05)$ is considered as statistically significant.

Methods The data so collected was entered into a computerised datasheet (Microsoft Excel 2007) and subjected to statistical analysis, using STATA 8 software.

Categorical data are expressed as numbers (Percentage) and continuous variable expressed as Mean (SD).

\section{Results}

In a 12 month period, 337 eyes were consecutively enrolled in the study. Both ultrasound A scan and Partial coherence interferometry was performed and IOL power implanted as per the IOL master.

Inclusion criterion and exclusion criterion used as mentioned.

The difference in between the final and predicted refraction is compared for both the groups.

Table 1

\begin{tabular}{|l|c|c|c|c|c|}
\hline \multicolumn{1}{|c|}{ Technique } & N & $\begin{array}{c}\text { Mean } \pm \text { SD } \\
\text { (MAE)* }\end{array}$ & Min. & Max. & p-value \\
\cline { 1 - 4 } A Scan & 337 & $0.402 \pm 0.407$ & 0 & 2.66 & \multirow{2}{*}{0.3802} \\
\hline IOL Master & 337 & $0.388 \pm 0.406$ & 0 & 2.41 & 0.380 \\
\hline
\end{tabular}

*MAE-Mean Absolute Error (Absolute value of MNE)

Table 2

\begin{tabular}{|l|c|c|c|c|c|}
\hline \multicolumn{1}{|c|}{ Technique } & N & $\begin{array}{c}\text { Mean } \pm \text { SD } \\
(\mathbf{M N E})^{*}\end{array}$ & Min. & Max. & p-value \\
\hline A Scan & 337 & $-0.226 \pm 0.526$ & -2.66 & 2.06 & \multirow{2}{*}{0.5148} \\
\hline IOL Master & 337 & $-0.217 \pm 0.518$ & -2.41 & 2.1 & 0.148 \\
\hline
\end{tabular}

*MNE-Mean numerical error (The difference between the refractive outcome and the predicted spherical equivalent)

The overall Mean Absolute error was $0.402 \pm 0.407$ with the ultrasound A scan \& $0.388 \pm 0.406$ for the IOL master, with the probability value (p-value) 0.3802 .

The Mean Numerical Error (the difference between the refractive outcome and the predicted spherical equivalent) is $-0.226 \pm 0.526$ ( $\min -2.66 \& \max 2.06$ ) for the ultrasound scan group and $-0.217 \pm 0.518$ (min -2.41 $\& \max 2.10$ ) for the IOL master group, with the probability value (p-value) being 0.514 .
We got a comparative result in our study, though not significant, but it is more in favour of IOL master (PCI) as per the mean absolute error and mean numerical error calculations.

When analysing the Mean Axial Length readings, the findings were almost similar, with an insignificant pvalue.

However the IOL master showed a less minimum and maximum values then the A scan.

Table 3: Mean axial length

\begin{tabular}{|l|c|c|c|c|c|}
\hline \multicolumn{1}{|c|}{ Technique } & N & Mean \pm SD & Min. & Max. & p-value \\
\hline A Scan & 337 & $23.66 \pm 1.88$ & 21 & 33.62 & \multirow{2}{*}{0.8312} \\
\hline IOL Master & 337 & $23.63 \pm 1.90$ & 20.93 & 33.74 & \multirow{2}{*}{ IOL } \\
\hline
\end{tabular}


In terms of refraction predictability according to different values of Axial Length (Mean \pm SD) of MNE,

Table 4

\begin{tabular}{|c|c|c|c|c|c|c|}
\hline Technique & \multicolumn{6}{|c|}{ Axial length-(Mean \pm SD) of MNE } \\
\hline & $\begin{array}{c}\left.\begin{array}{c}20-22 \\
(n 1=32 \\
\text { n2 }\end{array}=33\right)\end{array}$ & $\begin{array}{c}\left.\begin{array}{c}22-24 \\
(n 1=209 ; \\
\text { n2 }\end{array}=208\right)\end{array}$ & $\begin{array}{c}24-26 \\
(\mathrm{n} 1=69 ; \\
\text { n2 }=73)\end{array}$ & $\begin{array}{c}\begin{array}{c}26-28 \\
(\mathrm{n} 1=14 ; \\
\text { n2 }=11)\end{array}\end{array}$ & $\begin{array}{c}\left.\begin{array}{c}28-30 \\
(n 1=6 ; \\
\text { n2 }\end{array}=4\right)\end{array}$ & $\begin{array}{c}\text { Above30 } \\
\text { (n1=7; } \\
\text { n2=8) }\end{array}$ \\
\hline A Scan & $\begin{array}{c}-0.324 \pm \\
0.505\end{array}$ & $\begin{array}{c}-0.221 \pm \\
0.526\end{array}$ & $\begin{array}{c}-0.227 \pm \\
0.498\end{array}$ & $\begin{array}{c}-0.121 \pm \\
0.504\end{array}$ & $\begin{array}{c}-0.423 \pm \\
0.423\end{array}$ & $\begin{array}{c}0.064 \pm \\
0.936\end{array}$ \\
\hline $\begin{array}{l}\text { IOL } \\
\text { Master }\end{array}$ & $\begin{array}{c}-0.337 \pm \\
0.533\end{array}$ & $\begin{array}{c}-0.220 \pm \\
0.503\end{array}$ & $\begin{array}{c}-0.209 \pm \\
0.469\end{array}$ & $\begin{array}{c}-0.110 \pm \\
0.555\end{array}$ & $\begin{array}{c}-0.395 \pm \\
0.585\end{array}$ & $\begin{array}{c}0.204 \pm \\
0.971\end{array}$ \\
\hline p-value & 0.8904 & 0.7374 & 0.5913 & 0.9782 & 0.8312 & 0.4875 \\
\hline
\end{tabular}

Again the results were comparative with IOL master showing marginal improved accuracy over ultrasound in the $24 \mathrm{~mm}$ to $26 \mathrm{~mm}$ and $>26 \mathrm{~mm}$.
On seeing the cataract type the results were almost same, ultrasound showed improved accuracy in the denser cataract types (NS3/PSCC).

Table 5

\begin{tabular}{|l|c|c|c|c|}
\hline \multicolumn{1}{|c|}{ Technique } & \multicolumn{4}{|c|}{ Cataract type-(Mean \pm SD) of MNE } \\
\hline & $\begin{array}{c}\text { NS1 } \\
(\mathbf{n = 2 5 )}\end{array}$ & $\begin{array}{c}\mathbf{N S 2} \\
(\mathbf{n}=\mathbf{1 1 3})\end{array}$ & $\begin{array}{c}\mathbf{N S 3} \\
(\mathbf{n}=\mathbf{3 9})\end{array}$ & $\begin{array}{c}\mathbf{N S 4} \\
(\mathbf{n}=\mathbf{5})\end{array}$ \\
\hline A Scan & $-0.005 \pm 0.380$ & $-0.186 \pm 0.541$ & $-0.251 \pm 0.417$ & $-0.404 \pm 0.471$ \\
\hline IOL Master & $-0.007 \pm 0.454$ & $-0.191 \pm 0.515$ & $-0.254 \pm 0.398$ & $-0.466 \pm 0.481$ \\
\hline p-value & 0.9612 & 0.8915 & 0.8298 & 0.9158 \\
\hline
\end{tabular}

Table 6

\begin{tabular}{|l|c|c|c|}
\hline \multicolumn{1}{|c|}{ Technique } & \multicolumn{3}{|c|}{ Cataract type-(Mean \pm SD) of MNE } \\
\hline & $\begin{array}{c}\text { NS1PSCC } \\
(\mathbf{n}=\mathbf{5 3})\end{array}$ & $\begin{array}{c}\text { NS2PSCC } \\
(\mathbf{n}=\mathbf{8 4})\end{array}$ & $\begin{array}{c}\text { NS3PSCC } \\
(\mathbf{n}=\mathbf{1 8})\end{array}$ \\
\hline A Scan & $-0.343 \pm 0.575$ & $-0.224 \pm 0.572$ & $-0.339 \pm 0.368$ \\
\hline IOL Master & $-0.324 \pm 0.579$ & $-0.212 \pm 0.563$ & $-0.238 \pm 0.396$ \\
\hline p-value & 0.7020 & 0.7354 & 0.4103 \\
\hline
\end{tabular}

Mature and nearly mature cataracts were not included in the study.

\section{Discussion}

As cataract surgery has become a refractive procedure, the role of accurate intraocular power calculation is very important.

Most practices still use contact biometry and some immersion ultrasonic biometry.

Accurate biometry is the single most important factor for achieving successful refractive outcomes after lens implantation, even more important than different lens power calculation formulas. ${ }^{2}$

The techniques have always been technician dependent so good biometrists are invaluable and highly prized. The loss of such an individual often results in suboptimal predictions, all of which are problematic.

After minimal training, the partial coherence interferometry technology in the IOL master is reported to be as good as, possible better than, the best ultrasound technique.
We tried to validate this claim by comparing the results of partial coherence interferometry with those of the more common ultrasound contact biometry.

Many previous studies done have shown that the Partial Coherence Interferometry has significantly improved the refractive outcome with all 4 IOL power formulas. $^{3}$

Most of the studies done have compared IOL master with Immersion ultrasound, however in our study we compared Contact ultrasound and thus the factor of a trained biometrist come into play.

Julio Narvaez et $\mathrm{al}^{4}$ have compared both IOL master and Immersion ultrasound and shown equivalent results with both the modalities. They also stressed on the fact that ultrasound cannot be replaced, especially for eyes with dense media opacity.

But that was a study comprising of only 63 patients, in which 125 consecutive eyes were measured. In our study however, we have a large sample size of 337 patients, in which single eye was subjected to both the techniques and hence can predict more comprehensive result.

When comparing interobserver and intraobserver variability in IOL master measurements, Annette et $\mathrm{al}^{5}$ 
found that IOL master gives highly reliable and observer-independent measurements results.

Also if we compare the variable of operator experience in biometry measurements, Oliver et $\mathrm{al}^{6}$ has showed that the more experienced ones (having done more then 500 procedures) has less difference and lower variability in the difference between applanation ultrasound and IOL master readings.

This point is also been validated by our study, as our calculations were done by an experienced biometrist(done more than around 800 procedures) and it showed a comparative results in between the two, it points towards the impact of good training over a procedure.

And also as to how good it can predict the outcome of the procedure.

It has also been cited in previous studies that the applanation US results in measuring shorter axial length measurements. ${ }^{7}$ due to inadvertent indentation on corneal surface.

As pointed by R. Goyal et al ${ }^{1}$ ultrasound A scan is the most commonly used method for measuring axial length. An important limitation of this technique is that it requires that the ultrasound probe contacts the cornea, which can cause corneal indentation, thereby shortening the eye and leading to an underestimation of the true axial length. ${ }^{7}$

In their study the mean axial lengths as taken by the contact ultrasound technique and non-contact laser interferometry method were $23.35 \mathrm{~mm}$ (SD $1.81 \mathrm{~mm}$ ) and $23.55 \mathrm{~mm}$ (SD $1.76 \mathrm{~mm}$ ), respectively.

We also have approximately same results with the Mean and Standard deviation (+/-) is $23.66 \pm 1.88$ for A scan and $23.63 \pm 1.90$ for the IOL master.

The non contact method (Immersion ultrasound) though is less operator dependent. ${ }^{72}$

Study as done by Barbara et $\mathrm{al}^{8}$ have shown comparable results between the two modalities, which is also shown by our study.

They however compared Immersion ultrasound, which again point to the impact of experienced technicians in the measurement.

They have 45 patients as sample size, and in each patient they have randomly assigned one eye to IOL master and one to A scan, in contrast to our in which study single eye was subjected to both the procedures.

They have achieved a MAE (mean absolute error) of 2.0D in both the groups, in our study we have shown a MAE of less then 1.0D, which is even more indicative of precise biometry.

Study done by Simon Raymond et $\mathrm{al}^{4}$ has also compared MAE for both the techniques and found that there is no clinical advantage of IOL master over Applanation US in terms of refractive outcome.

They enrolled around 205 patients in their study and did a intention-to-treat analysis after randomization.

They have shown that the MAE in patients with implanted PCI-calculated IOLs was 0.40 - 0.37 D (SD;
95\% confidence interval $[\mathrm{CI}], 0.32-0.48 \mathrm{D})$ compared with $0.45-0.41 \mathrm{D}$ (SD; 95\% CI, 0.36-0.54 D) for patients with implanted AUS-calculated IOLs.

With no statistical significance between the two.

In our study we selected only those patients who were suitable for biometry by both the techniques, so there is no loss in randomization.

And even our results wasn't statistical significant, but they showed improved accuracy in the IOL master group.

R. Goyal et al $^{1}$ calculated the difference in axial length by both the modalities and showed that the A scan produced consistently low values then the IOL master.

Which therefore can produce biometry errors in IOL power calculations.

In our study however we took final spherical equivalent as the deciding factor, which is in turn suggestive of accuracy of axial length calculation.

They had a sample size of 100 patients which is even less then one third of our sample size.

We did not find any differences in refractive out comes comparing ultrasound with interferometry except for some small differences in post-operative refractive errors that were not clinically significant.

(p-value being 0.3802 and 0.5148 for Mean Absolute Error and Mean numerical Error, respectively)

The results for the eyes at the two extremes of normal distribution of axial length did not show any difference between the different methodologies. (p-value was not significant)

We found that the most significant limitation of interferometry was poor laser penetration in eyes with dense media opacities, especially with posterior subcapsular cataracts.

Also, because interferometry requires steady maintained fixation, it cannot be used when retinal disease or poor fixation is present. Nevertheless, on this basis we can conclude that interferometry cannot replace ultrasound biometry.

And, further trials are required to confirm whether the use of laser interferometry provides a consistent improvement in the accuracy of predicted postoperative emmetropia following cataract surgery.

\section{Summary}

1. Aim of the study was to compare optical biometry based on partial coherence laser interferometry (PCLI) principle to conventional ultrasound biometry in the accuracy of intraocular lens (IOL) power thereby to evaluate the predictability of refractive outcome using optical and ultrasound biometry.

2. We enrolled 337 patients during our study period who were willing to undergo cataract extraction and fit into our inclusion and exclusion criterion.

3. All the patients underwent a thorough pre-operative evaluation for cataract surgery and underwent a 
clear corneal temporal phacoemulsification (single surgeon, single lens, in the bag IOL placement).

4. ? Cases having major post-operative complications were not included in the study.

5. Preoperatively all the patients were subjected to both the biometry methods, i.e. 'Same Eye' was evaluated by ultrasound applanation biometry (Sonomed Inc.) and Partial Coherence Interferometry (IOL master. Zeiss Humphrey Systems)

6. For both the methods SRK T formula was used and predicted refraction (post-operative) as close to emmetropia as possible was determined, labelled as Expected / Predicted refraction.

7. Keratometry was also done using Bausch and Lomb keratometre.

8. IOL power based on both the methods were determined, and IOL was implanted according to the IOL master reading.

9. The study variables (for both the groups) were axial length measurement, IOL power calculation, Cataract type, Predicted refraction (Spherical equivalent), post-operative visual acuity at one month and Final refraction (Spherical Equivalent).

10. Mean Numerical Error (MNE) i.e. the difference between the refractive outcome and the predicted spherical equivalent and also Mean Absolute Error (MAE) i.e absolute value of MNE, were identified and designated as the main study outcome variable.

Following were the various outcomes of the study

1. The overall Mean Absolute error was $0.402 \pm 0.407$ with the ultrasound A scan \& $0.388 \pm 0.406$ for the IOL master, with the probability value ( $p$-value) 0.3802 .

2. The Mean Numerical Error (the difference between the refractive outcome and the predicted spherical equivalent) is $-0.226 \pm 0.526(\min -2.66 \& \max 2.06)$ for the ultrasound scan group and $-0.217 \pm 0.518$ (min -2.41 \& $\max 2.10$ ) for the IOL master group, with the probability value ( $\mathrm{p}$-value) being 0.514 .

3. On calculating the Mean axial length the ultrasound A scan showed 23.66 \pm 1.88 (Mean +-SD) and IOL master $23.63 \pm 1.90$, the minimum and maximum values we 21 and 33.62 for A scan and 20.93, 33.74 for the IOL master.

$\mathrm{P}$-value being insignificant (0.8312).

4. The MNE (mean numerical error) for different groups of axial length(in $\mathrm{mm}$ ) was calculated for both the procedures and it was as follows,

Axial Length (20-22) A scan -0.324 \pm 0.505

IOL Master $-0.337 \pm 0.533$

Axial Length (22-24) A scan -0.221 0.526

IOL Master $-0.220 \pm 0.503$

Axial Length (24-26) A scan -0.227 0.498

IOL Master $-0.209 \pm 0.469$

Axial Length (26-28) A scan -0.121 0.504

IOL Master $-0.110 \pm 0.555$

Axial Length (28-30) A scan -0.423 0.423
IOL Master $-0.396 \pm 0.585$

Axial Length (above 30) A scan 0.064 0.936

IOL Master 0.204 \pm 0.971

p-value being insignificant for all the groups.

5. The MNE was also calculated as per the cataract type, divided into 7 groups of NS1, NS2, NS3, NS4,

NS1 PSCC, NS2 PSCC, NS3 PSCC.

It was found out for both the A scan and the IOL master. The results were comparative but not significant.

\section{Conclusion}

When the first IOL (Intraocular lens) was implanted into an eye and found a surprise refractive error of -20 diopters (D) in 1949, there has been much work done to better the outcome of refractive status after cataract surgery.

Also with the improvements in surgical techniques and progress in the materials and designs of IOLs, patient expectations regarding post-operative refraction have also increased. So increased precision in the prediction of postoperative refraction is highly demanded and thus there is need of accurate Biometry in IOL power calculation.

A perfect refractive prediction requires good measurements of the corneal power, the axial length (AL), use of a suitable IOL calculation formula, an IOL with accurate power, and an uneventful surgery without any complications.

The basic difference between optical and acoustic techniques is due to different reflection sites at the retina. ultrasound measures up to the inner limiting membrane, whereas with partial coherence interferometry (PCI) the interference pattern from the retina is detected at retinal pigment epithelium. Furthermore, the misalignment of the beam axis and the visual axis during US measurements can cause a deviation in axial length measurements between optical and acoustic biometry methods.

In comparison with the ultrasound method, the main pre requisite for the accurate optical biometry method is that it needs a good fixation and clear media. It is accepted that it is less time consuming and more patient friendly than is ultrasound. However, it has a high failure rate, specifically in the presence of dense cataracts, which does not occur with ultrasound biometry. Although ultrasound biometry needs topical anesthesia and corneal applanation, it is able to measure AL in all eyes except those with intravitreous silicone oil.

The most revealing finding of our study is that we have excluded the cases in which IOL master was proven to be ineffective and then we calculated for both the groups simultaneously, then also we have got comparative results for both the techniques.

Also one more significant finding of our prospective study is that the calculation of IOL power based on ocular AL measurement using PCI technology provided no clinical advantage over conventional AUS, as measured by postoperative refractive outcome. It is 
likely that the apparent improvement of PCI over AUS reported in present studies will not be reinforced in future randomized clinical trials.

Another very valid point is that measurements by experienced operators showed less difference and lower variability in the difference between applanation US and IOL master readings for axial length. The noncontact optical method, which is essentially operator independent, some studies have also shown that PCI gives significantly more reliable biometry before cataract surgery, especially when performed by less experienced operators.

Also the PCI (IOL master) is a very simple and quick procedure, patients appreciate its simplicity \& comfort.

In the end, whatever said and done "Partial coherence interferometry cannot replace ultrasound biometry in day to day practice" as per now.

\section{References}

1. R. Goyal. Acta Opthalmol. Scand. 2003:81:331-335

2. Holladay JT, Prager TC, Ruiz RS, Lewis JW, Rosenthal $\mathrm{H}$. Improving the predictability of intraocular lens power calculations. Arch Ophthalmol. 1986;104:539-541.

3. Oliver Find. Improved prediction of intraocular lens power using partial coherence interferometry. J Cataract Refract Surg. 2001;27(6):861-7.

4. Simon Raymond. Comparing Ultrasound Biometry with Partial Coherence Interferometry for Intraocular Lens Power Calculations: A Randomized Study. Invest Ophthalmol Vis Sci. 2009;50(6):2547-52.

5. Vogel A, Dick HB \& Krummenauer F. Reproducibility of optical biometry using partial coherence interferometry: intraobserver and interobserver reliability. $J$ Cataract Refract Surg. 2001;27:1961-1968.

6. Seung Mo Kim. Refractive Predictability of Partial Coherence Interferometry and Factors that can Affect It Korean. 2009;23(1):6-12.

7. Hitzenberger CK. Optical measurement of the axial eye length by laser Doppler interferometry. Invest Ophthalmol Vis Sci. 1991;32:616-624.

8. Medical Sciences Advisory Committee. Optical Biometry Using Partial Coherence Interferometry Prior to Cataract Surgery. Canberra: Department of Health and Aging; 2005.

9. Retzlaff JA, Sanders DR \& Kraff MC. Development of the SRK/T intraocular lens implant power calculation formula. J Cataract Refract Surg. 1990;16:333-340.
10. Sanders DR, Retzlaff JA, Kraff MC, Gimbel HV \& Raanan MG. Comparison of the SRK/T formula and other theoretical and regression formulae. J Cataract Refract Surg. 1990;16:341-346.

11. Olsen T. Sources of error in intraocular lens power calculation. J Cataract Refract Surg. 1992;18:125-129.

12. Kalogeropoulos C, Aspiotis M, Stefaniotou M \& Psilas $K$. Factors influencing the accuracy of the SRK formula in the intraocular less power calculation. Doc Ophthalmol. 1994;85: 223-242.

13. Gantenbein. J fr Opthalmol. 2004;27(10):1121-1127.

14. Lam AK, Chan R \& Pang PC. The repeatability and accuracy of axial length and anterior chamber depth measurements from the IOL Master. Ophthalmic Physiol Opt. 2001;21:477-483.

15. Giers U \& Epple C. Comparison of A-scan device accuracy. J Cataract Refract Surg. 1990;16:235-242.

16. Verhulst E \& Vrijghem JC (2001): Accuracy of intraocular lens power calculations using the Zeiss IOL Master. A prospective study. Bull Soc Belge Ophtalmol. 2001;281:61-65.

17. Watson A \& Armstrong R. Contact or immersion technique for axial length measurement. Aust NZ J Ophthalmol. 1999;27:49-51.

18. Santodomingo-Rubido J, Mallen EA, Gilmartin B \& Wolffsohn JS. A new noncontact optical device for ocular biometry. Br J Ophthalmol. 2002;86:458-462.

19. Kim HJ, Kim HJ, Joo CK. Comparison of IOL Master, Ascan and Orbscan II for measurement of axial length and anteriorchamber depth. J Korean Ophthalmol Soc 2003;44:1519-27.

How to cite this article: Sharma A, Sharma A. V. Comparison of accuracy between ultrasound B scan and partial coherence interferometry (IOL master) in IOL (intraocular lens) power calculation. Indian $\mathrm{J}$ Clin Exp Ophthalmol. 2018;4(4):492-498. 